\title{
REFLEXIONES SOBRE LOS MOTIVOS DEL TRABAJO INFANTIL Y ADOLESCENTE DESDE LA ESFERA FAMILIAR EN MÉXICO*
}

\author{
SARAI MIRANDA JUÁREZ" \\ UNIVERSIDAD AUTÓNOMA DEL ESTADO DE MÉXICO
}

Recibido/Received/ Recebido: 23/08/2012 - Aceptado/ Accepted/ Aprovado: 27/03/2013

\begin{abstract}
Resumen
La investigación tuvo como propósito fundamental identificar los motivos por los cuales trabajan los niños y niñas ocupados en México. Se buscá generar una reflexión desde la perspectiva de las unidades domésticas como oferentes de fuerza de trabajo y al mismo tiempo como el espacio en que se concreta, organiza y materializa el trabajo de niños y niñas. La metodología utilizada fue cuantitativa, pues se revisán los datos arrojados por el Módulo de Trabajo Infantil incluido en la Encuesta Nacional de Ocupación y Empleo 2011. Se llega a la conclusión de que los niños y niñas ocupados en México lo hacen debido a dos causas principales: en primer lugar que en tanto unidades económicas productivas los hogares hacen uso del recurso fuerza de trabajo en la que los niños y las niñas, son actores centrales, y en segundo lugar por la insuficiencia de los ingresos de los hogares para sufragar los gastos personales y costos escolares.
\end{abstract}

Palabras clave: Trabajo infantil, Estudios demográficos, Empleo en México.

\section{REFLECTIONS ON THE INCENTIVES OF CHILD AND ADOLESCENT WORK FROM THE FAMILIAR ENVIRONMENT IN MEXICO}

\begin{abstract}
This article aims to describe the main reason why children work in Mexico. It seeks to generate reflection from the household perspective as suppliers of workforce and at the same time, as the specific place, where children's work is organized and materialized. The methodology is quantitative; as we review the data obtained from the child labor module included in the National Survey of Occupation and Employment 2011. The conclusion is that the reason for children to work in Mexico it is basically due to two main factors; first, that productive economic units such as households use children as workforce resources being them the main actors and second, because the existence of insufficient household income to cover personal expenses and school fees.
\end{abstract}

Keywords: Child labor, Demographic studies, Employment in Mexico.

Investigación realizada a partir de la revisión de la principal fuente de datos estadísticos sobre trabajo infantil en México, el Módulo de Trabajo Infantil de la Encuesta Nacional de Ocupación y Empleo 2011.

* Economista de la Universidad Autónoma del Estado de México, Maestra en Ciencias Sociales con Especialidad en Desarrollo Municipal del Colegio Mexiquense y doctora en Estudios de Población del Colegio de México. Docente de la Universidad Autónoma del Estado de México. Correo electrónico: saraimirandaj@gmail.com 


\title{
REFLEXÕES SOBRE OS MOTIVOS DO TRABALHO INFANTIL E ADOLESCENTE A PARTIR DA ESFERA FAMILIAR NO MÉXICO
}

\section{Resumo}

\begin{abstract}
O presente artigo tem como propósito central descrever os motivos pelos quais, no México, meninos e meninas trabalham. Procura-se gerar uma reflexão desde a perspectiva das unidades domésticas como oferentes de força de trabalho e ao mesmo tempo como o espaço em que se concretiza, se organiza e se materializa o trabalho de meninos e meninas. A metodologia utilizada é quantitativa, pois se revisam os dados encontrados pelo Módulo de Trabalho Infantil da Pesquisa Nacional de Ocupação e Emprego de 2011. Chega-se à conclusão de que os meninos e as meninas ocupados no México trabalham devido a duas causas principais: em primeiro lugar porque como unidades econômicas produtivas os lares fazem uso do recurso da força de trabalho na que os meninos e as meninas são atores centrais, e em segundo lugar pelos insuficientes recursos dos lares para atender aos gastos pessoais e custos escolares.
\end{abstract}

Palavras chave: Trabalho infantil, Estudos demográficos, o Emprego no México.

Miranda, S. (2013) Reflexiones sobre los motivos del trabajo infantil y adolescente desde la esfera familiar en México. En: Revista de la Facultad de Ciencias Económicas de la Universidad Militar Nueva Granada. rev.fac.cienc.econ, XXI (1)

JEL: J21, J82.

\section{Introducción}

“(...) uno quisiera que los hijos se dedicaran siempre a estudiar pa que fueran algo de importancia, pero no alcanza, porque hay veces que uno se pone a pensar en la vida que nosotros vivimos, de que la secundaria estaba lejos y nuestros papás no tenían, así pa mandarnos, no tenían recursos pa mandarnos, ahora si como quien dice aunque nosotros hayamos querido estudiar, no se pudo, el mío está en segundo y se me hace que este año ya no va, son muchos gastos de cooperaciones, que un día 500, que otro 100, según es gratis, pero ¿cuál gratis? puros gastos (...)"1

El trabajo infantil es un fenómeno social que ha preocupado tanto al sector académico como al sector gubernamental. Se han buscado siempre las razones y motivos por los que los niños y las niñas trabajan con el propósito de proponer acciones a fin de disminuir la participación de los niños en las actividades productivas. Los datos más recientes, provenientes del Módulo de Trabajo Infantil de la Encuesta Nacional de Empleo de 2011 muestran que en México existen poco más de tres millones de razones (3.035.364 niños y niñas que se declaran ocupados) para escudriñar sobre los motivos que están detrás de esta práctica.

Dicho fenómeno se ha estudiado desde varias perspectivas, económica, antropológica, sociológica, y desde estos diferentes puntos de vista se ha llegado a una conclusión común: el trabajo infantil sería parcialmente comprendido si se analiza sólo a través de las condiciones generales que se presentan en el mercado de trabajo. La dotación de niños y niñas al mercado de trabajo proveniente de las unidades familiares, es un factor relevante para completar la fotografía del fenómeno estudiado. Este artículo versa precisamente sobre los principales motivos de la práctica del trabajo infantil, partiendo del supuesto de que, es desde el ámbito doméstico

1 Testimonio de un padre de familia, pequeño productor agrícola. 
donde se abastece de mano de obra familiar a los mercados de trabajo que así lo requieren.

En la literatura sobre las tendencias más actuales de los mercados de trabajo, existe una importante corriente que parte del reconocimiento de que las unidades domésticas ${ }^{2}$ tienen un papel dual. En primer lugar, ponen en práctica estrategias de diversos tipos, para garantizar la supervivencia económica (entre ellos la movilización de la mayoría de los miembros de la familia como fuerza de trabajo); y por otra parte, la unidad doméstica funciona como un ente mediador frente a las condiciones estructurales que imperan en el entorno social y económico (Villasmil, 1998).

Bajo esta premisa, se refleja la necesidad de considerar que "cada una de las unidades domésticas ponen en práctica mecanismos de reproducción particulares, según los recursos materiales de que disponen y el tipo de intensidad de presiones externas" (Bronfman, Lerner \& Tuirán, en Villasmil, 1998, 70). En este sentido, la oferta de niños y niñas al mercado de trabajo, constituiría una estrategia por parte de las unidades domésticas para hacer frente a las presiones económicas que impone el contexto socioeconómico y que, en las últimas décadas, se expresa en sucesivas crisis económicas que han llevado al despliegue de diversas acciones por parte de los grupos domésticos. Esto último, con el fin de asegurar la reproducción material cotidiana.

Cabe destacar, que el fenómeno de producciónreproducción en el cual se inscribe la lógica de las unidades domésticas, en particular aquellas que se encuentran en situación de desventaja material y económica no es algo nuevo, ya los clásicos como Marx y Chayanov habían profundizado al respecto. No obstante, en este artículo la preocupación gira en torno al papel de la unidad doméstica como proveedora de fuerza de trabajo infantil en el mercado laboral. Resulta, entonces, relevante escudriñar los principales motivos que despliegan la unidad doméstica para la entrada de los menores a los mercados de trabajo.

Si bien el uso de niños en los mercados laborales tiene como uno de sus principales orígenes el deterioro de las condiciones de vida de las familias; hay también factores derivados del lado de la demanda tales como la presencia de mercados de trabajo que reclaman este tipo de mano de obra. Es decir, hay elementos combinados de oferta y demanda que pueden explicar la prevalencia del trabajo infantil. Sin embargo, el objetivo central de este artículo es conocer los principales motivos del trabajo infantil únicamente desde el ámbito de los hogares. Para ello se hace un análisis de los datos que ofrece el módulo de trabajo infantil de la Encuesta Nacional de Ocupación y Empleo del año 2011.

La metodología a utilizar es básicamente descriptiva pues se realiza una revisión de los motivos para trabajar de cada niño y niñas por edad, sexo y sector de actividad. Se considera que este ejercicio aunque modesto, constituye un importante paso para explicar un fragmento del complejo fenómeno del trabajo infantil en México.

\section{El trabajo infantil y adolescente en la esfera doméstica}

El trabajo infantil y adolescente puede ser analizado desde el enfoque de las estrategias de vida, que representa uno de los esfuerzos más integrales en el intento de aprehender las prácticas de los grupos sociales en situación de escasez material. Por ello, uno de los elementos centrales en esta perspectiva

2 Existe una amplia discusión respecto a las ambigüedades que presenta el concepto de unidad doméstica. Se ha definido de indistintas maneras considerando aspectos como ingresos y gastos en común (Barsotti, 1981). De hecho, esto se refleja en las diferentes "(...) definiciones censales: proveer sus necesidades alimenticias y otras esenciales para vivir, cocinar y comer en conjunto (Argentina) vivir juntos y compartir al menos una comida diaria (Barbados); hacer vida en común bajo un régimen familiar, compartir la vivienda y tomar juntos sus comidas (Costa Rica); vivir juntos compartir, "common living arragements", pueden compartir o no sus comidas (Jamaica); vivir bajo un régimen familiar o parecido (Paraguay) (Barsotti,1981)). De estas definiciones queda en evidencia que el concepto de unidad doméstica no coincide con el de la unidad familiar definida según el parentesco, el matrimonio o la residencia" (Barsotti, 1981, 177). 
es el reconocimiento de las unidades domésticas que ejercen un papel de mediadoras entre los procesos macro sociales y los individuos. Como afirma Villasmil (1998, 71) “(...) cobra relevancia el abordaje de la unidad doméstica como unidad de análisis, toda vez que es dentro de ésta que se gestan los procesos de producción inter generacional, y en consecuencia, donde se decide la participación económica familiar como componente esencial de dichas estrategias". En este caso, las unidades domésticas son las entidades que proveen fuerza de trabajo de niños y niñas al mercado y reciben de éste ya sea beneficios o desventajas.

Hay quienes se refieren a la familia, al hogar o bien a la unidad doméstica, como la principal instancia mediadora y existe un reconocimiento implícito de que es en la familia donde suceden la mayoría de las prácticas y se materializan los efectos de las estrategias puestas en marcha. Como afirma González de la Rocha $(2009,12)$ :

\section{“(..) los grupos domésticos (...) son los escenarios de sobrevivencia, en donde se instrumentan prácticas y mecanismos para enfrentar y sobrellevar la vida en pobreza y los problemas que la escasez de recursos acarrea en la vida cotidiana de los individuos. Es ahí, en el seno de la vida en familia, con sus elementos de cohesión y unidad pero, también, con to- das sus desarmonías, fisuras y desigual- dades, donde los sujetos de nuestro es- tudio luchan día con día para asegurar el acceso a bienes y servicios básicos para sobrevivir en contextos en donde el em- pleo no siempre es suficiente o posible" (González de la Rocha, 2009, 12).}

En este contexto, resulta ineludible ver "a las unidades familiares en cuanto unidades de decisión $y$ de recursos en la organización social de la reproducción de los agentes sociales" (Barsotti, 1981, 177). De lo contrario sería imposible entender el fenómeno de la reproducción social y de la reproducción de situaciones de desventaja, además se dejaría de lado la importancia de procesos tales como las decisiones económicas, la organización del trabajo doméstico y la participación en el mercado de trabajo que se materializan dentro de las unidades familiares (Barsotti, 1981).

Por ello, uno de los aportes de la perspectiva teórica de las estrategias de sobrevivencia es el reconocimiento de que los hogares, las familias o las unidades domésticas son una fuente tanto de recursos como de decisiones. Para Barsotti (1981) las unidades familiares se pueden considerar desde dos puntos de vista: como unidades de decisión y como unidades de recursos.

Para Barsotti, las unidades familiares pueden considerarse como unidades de decisión, debido a que es en este escenario en dónde se determinan las características del proceso de reproducción de los agentes pertenecientes a la unidad familiar; en tanto que pueden ser consideradas como unidades de recursos ya que es mediante éstos que les es posible ejercer su responsabilidad en la reproducción de los agentes sociales (Barsotti, 1981). Coincidentemente, con lo anterior González de la Rocha establece que:

"El grupo doméstico ha sido conceptualizado como una instancia mediadora entre los individuos y la estructura socioeconómica, como escenario de procesos de toma de decisiones -no libres de conflicto y negociación- y de puesta en práctica de acciones y respuestas que se instrumentan ante los cambios del mercado de trabajo y los sistemas productivos más amplios (González de la Rocha, 2009, 48)".

Con lo que se refuerza la idea de que las unidades familiares, representan la instancia en la que se movilizan y organizan los recursos para el logro de ciertos objetivos referidos a la unidad o a sus miembros, cualquiera que sea el grado de conciencia que éstos tengan acerca de esa organización y esos objetivos (Barsotti, 1981).

Como un refuerzo a la idea anterior Sáenz \& Di Paula (1981) establecen que el enfoque de las estrategias de supervivencia analiza los mecanismos y procesos 
desde el punto de vista del agregado familiar o unidad doméstica, debido a que es precisamente en ésta en donde se presentan la mayor parte de las variables vinculadas a la producción, consumo y reproducción de la fuerza de trabajo en los sectores de menores recursos:

"El agregado familiar, explícita, consciente, en forma planificada o no planificada adopta, entre las alternativas disponibles en el modelo de desarrollo determinado, las que consideran más apropiadas para satisfacer sus necesidades relativas pudiendo crear contenidos específicos en los componentes usados" (Sáenz \& Di Paula, 1981, 152-153).

Así, al parecer en las unidades domésticas se generan estrategias "con componentes dados en la sociedad global que son "elegidos" por las familias, otras con instancias "creadas" en el ámbito familiar y otras "inducidas" en agregados mayores que el familiar pero menores que el de la sociedad global $y$ que son utilizados por la familia con mayor eficiencia" (Sáenz \& Di Paula, 1981, 152-153). Un ejemplo de ello es la hipótesis ampliamente trabajada por García \& De Oliveira (2006) en la que reconocen que es precisamente en la unidad doméstica en donde se presenta una fuerte influencia en la formación de la oferta de fuerza de trabajo, debido a que es en los hogares dónde se posee el recurso de individuos disponibles para la actividad económica y que tanto el número como las características de quienes participan en el mercado de trabajo depende en gran medida de la composición de los grupos domésticos. Con esto, se deja ver una evidente forma de intermediación del grupo doméstico entre los individuos que trabajan y los mercados laborales.

En este mismo tenor, pero desde un punto de vista más enfocado a la reproducción de la fuerza de trabajo, Susana Torrado (1981) afirma que es mediante la utilización de la familia que se asegura la "oferta de trabajadores libres" y por tanto se asegura la reproducción tanto de la fuerza de trabajo como la del "trabajador libre", estableciendo que:
“(...) la reproducción de la fuerza de trabajo se verifica en la unidad familiar mediante el cumplimiento de las tareas indispensables para la reconstitución y mantenimiento de la fuerza de trabajo activa, así como de aquéllas que aseguran el reemplazo generacional de los trabajadores a través de la creación, sustento y educación de su progenitura (...) La aparición en el mercado del empleo de la fuerza de trabajo así creada bajo la forma de "trabajador libre" se asegura a través de las normas jurídicas que regulan el ejercicio de autoridad dentro del grupo, particularmente en lo que se relaciona con el uso de la fuerza de trabajo disponible dentro de la unidad familiar. En tal sentido, la legislación relativa a la "mayoría de edad" que libera a los hijos de toda obligación económica hacia sus progenitores en el momento en que alcanzan el umbral productivo, fija una de las principales modalidades de aparición del "trabajador libre" dentro de las poblaciones ya incorporadas a la esfera de producción capitalista" (Torrado, 1981, 217).

Para Torrado (1981) la unidad familiar actúa como mediadora entre los individuos trabajadores $y$ los requerimientos del capital económico, por un lado como generadora de "trabajadores libres" obligados a vender su fuerza de trabajo como mercancía y por otro como una instancia que comprende "los mecanismos utilizados para sufragar el costo de reproducción de la fuerza de trabajo minimizando la parte de ese costo que es asumida por el capital" (Torrado, 1981, 217).

Otro importante acercamiento al estudio de las unidades domésticas en situación de pobreza y desventaja material son las aportaciones teóricas y metodológicas de Daniel Bertaux quien concibe a las unidades domésticas o familias como "microsistemas auto-poieticos orientados a la producción de energías humanas de sus propios 
miembros, tanto en la vida cotidiana como a largo plazo" (Bertaux, 1996, 12). Desde esta perspectiva, las unidades domésticas se organizan en función de una lógica apegada a las emociones y sentimientos, es decir, la "lógica del regalo", que la hace diferente a la lógica mercantil; así, para Bertaux "las relaciones intrafamiliares se caracterizan por el esfuerzo y la solidaridad de los miembros con el objetivo de hacer posible la subsistencia común" (Macri, 2011, 726).

Un aporte analítico de suma importancia en los trabajos de Bertaux (1996) es el reconocimiento de que las unidades domésticas son también sistemas abiertos con los medios que las rodean y en este sentido sus mecanismos de auto regulación, auto organización auto orientación y auto diferenciación pueden ir acompañados de acumulación de recursos o bien acumulación de desventajas. Así, el enfoque de las estrategias de reproducción y supervivencia resulta adecuado para enfrentar el estudio de los fenómenos sociales a través de una instancia mediadora, en este caso la unidad doméstica, que vincula los fenómenos con los niveles de análisis microsocial y macrosocial, mediante la puesta en práctica de acciones que pueden ser o no conscientes, pero que permiten que se presenten procesos de reproducción y supervivencia.

Diversas reflexiones surgen al haber revisado la línea de investigación expuesta. En primer lugar que el trabajo infantil forma parte del comportamiento estratégico de las unidades domésticas y tiene como propósito asegurar la reproducción biológica y material, y con ello, se van configurando las formas de inserción laboral y social de los hijos e hijas. En segundo lugar, que las estrategias de reproducción y supervivencia están permeadas por contextos externos con los que se articulan las prácticas de dichas unidades.

$\mathrm{Al}$ parecer las unidades domésticas organizan sus recursos y los hacen variar para adaptarse a las exigencias y necesidades de la sociedad en la que están inmersas, adoptan estrategias de acción en un nuevo entorno para perseguir un objetivo: su supervivencia. Así, desde la esfera doméstica, es preciso puntualizar en la paradoja a la que se enfrentan las familias. En la búsqueda de su supervivencia hacen uso de estrategias a corto plazo con objeto de resarcir eventos inesperados o situaciones críticas que pudieran amenazar la subsistencia:

"Frente a situaciones críticas (...), en que un segmento o capas enteras de la sociedad ven empeorar o deteriorarse sus niveles de vida, llegando a sentir seriamente amenazada su reproducción material y biológica es cuando tiene sentido, (...) hablar de "estrategias de supervivencia" como fenómeno social, en tanto estas capas desarrollan un complejo de conductas con el objeto de "resistir" a las fuerzas o procesos deteriorantes a que están sometidas. Que esto sea así no significa necesariamente que tales "respuestas" o "readaptaciones" sean exitosas, puesto que la incapacidad crónica de una economía para insumir trabajo productivo puede hacer estéril o ineficaz cualquier estrategia” (Valdés \& Acuña, 1981, 235).

En este tenor, en situaciones de crisis los hogares se ven inducidos a la adopción de estrategias que como bien aduce González de la Rocha (2009) resultan ser de dos caras, ya que cuando las unidades domésticas se enfrentan a un deterioro de su condiciones de existencia, una de las principales reacciones de los hogares es la colocación de más miembros en el mercado de trabajo, incluyendo la incorporación de mujeres y niños, en los mercados laborales que se ofrezcan en el contexto determinado. Por lo que la entrada de nuevos miembros al mercado laboral puede ir desde el trabajo familiar no remunerado, el acceso al mercado informal, así como la inserción en mercados caracterizados por condiciones flexibilizadas y precarias.

De hecho "el trabajo de los niños, en contextos de extrema precariedad (...), parece ser un recurso que se invierte en estrategias de supervivencia a corto plazo" (González de la Rocha, 2009, 60). Las estrategias de sustento se encuentran vinculadas con la movilización de la fuerza de trabajo, pues éste es uno de los recursos fundamentales con que 
cuentan los hogares. Ahora bien, una vez expuesta la revisión conceptual respecto al ámbito doméstico y su papel en la práctica del trabajo infantil, se hace necesario revisar los datos empíricos al respecto. Lo cual se presenta en la siguiente sección.

\section{Breve descripción del trabajo infantil y adolescente en México}

A continuación se presenta un análisis descriptivo de los datos que proporciona el Módulo de trabajo infantil perteneciente a la ENOE $2011^{3}$. Los datos más recientes sobre el trabajo infantil en México muestran a nivel nacional existen 28.886 .326 personas de 5 a 17 años, de ellas 25.850 .962 dijeron no trabajar mientas que 3.035.364 se encuentran ocupadas en alguna actividad económica, lo que equivale a $11 \%$ del total de dicha población (Ilustración 1).

Ilustración 1. Condición de ocupación de la población de 5 a 17 años en México (\%) ${ }^{4}$

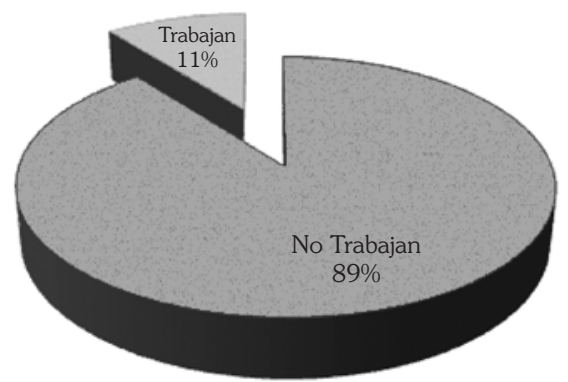

Desglosando la información por sexo se observa que de las 3.035.364 personas de 5 a 17 años que se encuentran ocupadas en alguna actividad económica 2.062.506 son hombres y 972.858 mujeres, es decir 68\% niños y 33,2\% niñas. La tasa de participación de los niños es más alta que de la de las niñas, 14\% de los niños dijeron estar ocupados, mientras que para del total de las niñas de ese mismo grupo de edad se calculó una tasa de $7 \%$ (Ilustración 2). Este primer acercamiento pone en evidencia que desde una observación global, la participación laboral de los varones duplica la participación de las mujeres. En este sentido, un niño que oscila entre los 5 y 17 años de edad tiene el doble de probabilidad de trabajar que una niña entre estas mismas edades.

Algunos estudios a nivel de América Latina han mostrado cómo la participación laboral de los menores varones aventaja por mucho a la participación laboral de las niñas (Alarcón, 2006). Tal situación podría estar explicada por múltiples factores pero en general la literatura especializada alude a la fuerte tendencia sobre la situación de los niños y las niñas para ir delimitando sus roles sociales a partir de criterios de género, lo cual se conjuga con un proceso de disminución de la tasa de ocupación femenina en estas edades como resultado de su ocupación en tareas domésticas de su propio hogar. Estas labores históricamente han sido invisibilizadas y escasamente declaradas (Alarcón, 2009).

Ilustración 2. Población de 5 a 17 años ocupada desplegada por sexo $(\%)^{5}$

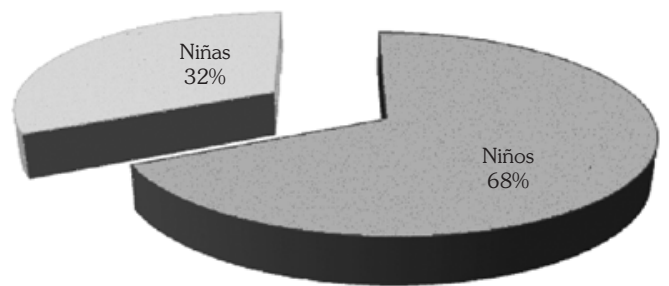

Asimismo, si se desglosa la información por edad, es posible observar que la mayor cantidad de niños $y$ niñas que trabajan va en aumento a medida que

3 Cabe señalar que el cuestionario del Módulo de Trabajo Infantil es aplicado a niños y niñas bajo la supervisión de un adulto del hogar, ello podría estarse reflejando en el resultado global sobre las dimensiones del trabajo infantil y adolescente en México ya que la presencia de un adulto podría influenciar las repuestas. No obstante, esta problemática excede los límites que corresponden a las reflexiones que se presentan en este artículo.

4 Elaboración propia con base en Encuesta Nacional de Ocupación y Empleo 2011, Módulo de Trabajo Infantil, INEGI (2012).

5 Elaboración propia con base en Encuesta Nacional de Ocupación y Empleo 2011, Módulo de Trabajo Infantil, INEGI (2012). 
aumenta la edad. Ello se muestra en la Ilustración 3 que contiene las tasas de participación laboral por edad. Evidentemente hay una relación entre mayor edad y mayor tasa de ocupación. Ello probablemente está sugiriendo que al interior de los hogares es el grupo de los hijos e hijas de más edad son los que se incorporan a las actividades económicas. Sin embargo también puede estar oculto y subestimado el trabajo doméstico de los niños y niñas más pequeñas que van sustituyendo a los hermanos mayores con lo que se facilita la incorporación al trabajo o a la escuela de los mayores.

Otro dato útil al respecto es que la edad media a la entrada del primer trabajo es de 8 años. Lo que significa que en México los niños y niñas ocupados inician su actividad laboral en promedio a los 8 años de edad.

Ilustración 3. Tasa de participación laboral de la población de 5 a 17 años ocupada, desplegada por edad (\%) ${ }^{6}$

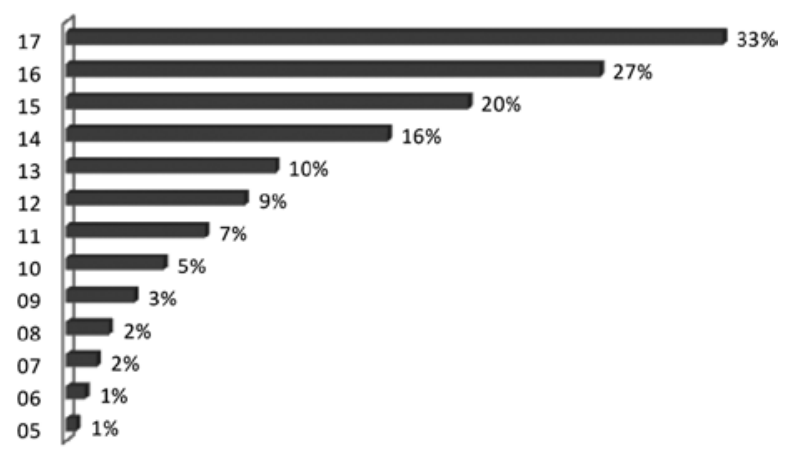

Los datos muestran también la distribución de quienes trabajan por sector de actividad entre niños y niñas. En términos globales sale a la luz que es el sector comercio y servicios en donde se ocupa la mayor parte de esta población (52\%), le sigue el sector agropecuario (29\%), y finalmente industria y construcción (17\%). Si este análisis se realiza desplegado por sexo, los resultados cambian, para el caso de los niños por ejemplo, se tiene que cuatro de cada diez niños varones ocupados (38\%) realiza labores agropecuarias, en tanto que para el caso de las niñas se registra que siete de cada diez niñas ocupadas (73\%), se emplean en el sector servicios ya sea como comerciantes en el sector informal o empleadas en comercios establecidos. Cabe señalar también que $18 \%$, de los niños se desempeñan en trabajos relativos a la industria y las artesanías o bien se registran como ayudantes en este último sector (ENOE 2011, INEGI; 2012).

\section{Los motivos del trabajo infantil y adolescente en México}

Se sabe que la pobreza y las necesidades económicas son las principales causas de la existencia del trabajo infantil, aunque más allá de ello, pueden presentarse múltiples razones. La connotación del trabajo infantil es diferente en distintos contextos socioeconómicos y culturales. Estudios recientes revelan que los padres encuentran benéfico el trabajo de los niños y las niñas para el sano desarrollo de éstos. Los sitúa frente a responsabilidades y los ayuda a madurar. En cierta medida les genera una experiencia de aprendizaje que difícilmente podrían obtener de otros contextos como el escolar, el familiar y el recreativo (Alarcón, 2009).

Hay también algunas hipótesis respecto al trabajo como una importante alternativa al ocio, ya que los padres y/o los jefes y jefas de los hogares consideran que desde edades tempranas los niños y las niñas deben aprender formas de ganarse la vida y el sustento cotidiano. Asimismo, otra de las hipótesis extendidas es que una de las mejores herencias que puede dejarse a los hijos e hijas es el aprendizaje temprano de un oficio o actividad laboral que les asegure una forma de sustento en el futuro (Alarcón, 2006).

Respecto a los motivos por los que trabajan las personas de este grupo de edad que proporciona el Módulo de Trabajo infantil de la ENOE 2011 se observa que existen varias razones. La razón "el hogar necesita de su trabajo" es la que mayor porcentaje de respuestas presenta (28\%), le sigue "pagar gastos de escuela y gastos propios" (26\%). El siguiente motivo más mencionado fue "aprender un oficio" con 16\%, mientras que "el hogar necesita de su aportación económica" y

6 Fuente: Elaboración propia con base en Encuesta Nacional de Ocupación y Empleo 2011, Módulo de Trabajo Infantil, INEGI (2012). 
"no quiere ir a la escuela" aparecen en último lugar con $13 \%$ y $5 \%$ respectivamente (Tabla 1 ).

Tabla 1. Porcentaje de población ocupada 5 a 17 años desplegada por sexo y motivos por los que trabaja ${ }^{7}$

\begin{tabular}{|c|c|c|c|}
\hline & Niños & Niñas & Total \\
\hline $\begin{array}{l}\text { El hogar necesita de su } \\
\text { aportación económica }\end{array}$ & $69 \%$ & $31 \%$ & 390432 \\
\hline El bogar necesita de su trabajo & $65 \%$ & $35 \%$ & 836794 \\
\hline Aprender un oficio & $79 \%$ & $21 \%$ & 464737 \\
\hline $\begin{array}{l}\text { Para pagar su escuela y sus } \\
\text { propios gastos }\end{array}$ & $65 \%$ & $35 \%$ & 769128 \\
\hline No quiere ir a la escuela & $83 \%$ & $17 \%$ & 148403 \\
\hline Otra razón & $59 \%$ & $41 \%$ & 373714 \\
\hline Total & $68 \%$ & $32 \%$ & 2983208 \\
\hline
\end{tabular}

$\mathrm{Al}$ escudriñar los motivos por los que trabajan entre niños y niñas se observan algunas diferencias. En el mismo Tabla resalta por ejemplo, que para las niñas es mayor el porcentaje en los motivos "el hogar necesita de su trabajo" y "el pago de la escuela y gastos propios". Estos datos podrían estar sugiriendo que para el caso de las niñas hay menores posibilidades de que la familia sufrague los gastos relacionados con la escolaridad. Cabe resaltar que para familias en situación de escases material o pobreza, que los hijos e hijas vayan a la escuela supone un gran esfuerzo "la educación pública aunque gratuita es, en realidad, demasiado cara para una familia pobre que debe adquirir los libros y los otros materiales escolares, los uniformes, la ropa, el transporte...en muchos lugares, el mantenimiento de un hijo en la escuela puede llegar a representar un tercio de los ingresos totales en efectivo de las familias y muchas de éstas tienen más de un hijo en edad escolar" (Intervida, 2008).

Aunado a ello también se observa que la motivación "aprender un oficio" es más alta en los niños que en la niñas. Estos comportamientos al parecer están indicando que la participación de los niños y las niñas en el trabajo responde en cierta medida a eventos vinculados con los roles de género. Para los niños el aprendizaje temprano de un oficio es mucho más importante que para las niñas, en tanto que para las niñas es más importante el hecho de que "el hogar necesita de su trabajo". Lo que es un factor que también llama la atención es que para los niños ser proveedores tempranos es un motivo que tiene particular importancia, ya que el porcentaje para el motivo "el hogar necesita de su aportación económica" es de 65\%, frente a un 35\% de las niñas.

Desplegando la información por grupos de edad se pueden observar también algunas particularidades, por ejemplo, para los niños más pequeños, de 5 a 9 años de edad, el motivo que registra mayor número de respuestas es "otra razón" que puede incluir un infinito rango de opciones (Tabla 2). No obstante, lo que se sabe al respecto, es que los niños y niñas de menor edad que participan en algún trabajo lo hacen por lo regular en la lógica del acompañamiento de algún otro miembro del hogar, pues al ser los más pequeños los hogares se enfrentan al escaso acceso a servicios de cuidado de niñas y niños menores durante el tiempo en que los adultos realizan actividades económicas (Alarcón, 2009).

Se sabe también que el trabajo de los niños y niñas en este grupo de edad puede responder a las necesidades de trabajo doméstico que se presentan cuando otros miembros del hogar se incorporan al mercado de trabajo o a otras actividades extra domésticas. En el Tabla 2 se observa que 26\% de los niños y niñas de 5 a 9 años de edad trabajan debido a que en el hogar se necesita de su trabajo.

Tabla 2. Motivos por los que trabaja la población ocupada 5 a 9 años desplegada por sexo ${ }^{8}$

\begin{tabular}{|c|c|c|c|c|}
\hline & Niños & Niñas & Total & $\%$ \\
\hline $\begin{array}{l}\text { El bogar necesita de su } \\
\text { aportación económica }\end{array}$ & 2189 & 334 & 2523 & $1 \%$ \\
\hline $\begin{array}{l}\text { El bogar necesita de su } \\
\text { trabajo }\end{array}$ & 31324 & 16185 & 47509 & $26 \%$ \\
\hline Aprender un oficio & 23399 & 14072 & 37471 & $20 \%$ \\
\hline $\begin{array}{l}\text { Para pagar su escuela y } \\
\text { sus propiso gastos }\end{array}$ & 16439 & 11266 & 27705 & $15 \%$ \\
\hline No quiere ir a la escuela & o & o & o & $0 \%$ \\
\hline Otra razeón & 40892 & 29305 & 70197 & $38 \%$ \\
\hline Total & 114243 & 71162 & 185405 & $100 \%$ \\
\hline
\end{tabular}

7 Elaboración propia con base en Encuesta Nacional de Ocupación y Empleo 2011, Módulo de Trabajo Infantil, INEGI (2012).

8 Elaboración propia con base en Encuesta Nacional de Ocupación y Empleo 2011, Módulo de Trabajo Infantil, INEGI (2012). 
Para el grupo niños en edad de 10 a 13 años se observa igualmente que el motivo principal es que "el hogar necesita de su trabajo" con 35\%, siguiéndole el motivo "aprender un oficio" con 23\%. La literatura especializada sobre trabajo infantil ha puesto de manifiesto que los hogares en situación de pobreza buscan que los hijos y las hijas se entrenen en algún oficio que les permita enfrentar laboralmente el futuro pues la educación formal no constituye una opción en la que se vea un mecanismo de movilidad social ascendente (Intervida, 2008), al parecer en México la lógica de los hogares está en esa misma dirección, sobre todo cuando va en aumento la edad en que se encuentran los niños y niñas (Tabla 3).

Tabla 3. Motivos por los que trabaja la población ocupada 10 a 13 años desplegada por sexo ${ }^{9}$

\begin{tabular}{|c|c|c|c|c|}
\hline & Niños & Niñas & Total & $\%$ \\
\hline $\begin{array}{l}\text { El bogar necesita de su } \\
\text { aportación económica }\end{array}$ & 35607 & 9935 & 45542 & $7 \%$ \\
\hline $\begin{array}{l}\text { El bogar necesita de } s u \\
\text { trabajo }\end{array}$ & 161256 & 83738 & 244994 & $35 \%$ \\
\hline Aprender un oficio & 122547 & 36800 & 159347 & $23 \%$ \\
\hline $\begin{array}{l}\text { Para pagar su escuela y } \\
\text { sus propiso gastos }\end{array}$ & 81684 & 46092 & 127776 & $18 \%$ \\
\hline No quiere ir a la escuela & 10473 & 3685 & 14158 & $2 \%$ \\
\hline Otra razón & 61589 & 43967 & 105556 & $15 \%$ \\
\hline Total & 473156 & 224217 & 697373 & $100 \%$ \\
\hline
\end{tabular}

En el Tabla 4 se muestra que los adolescentes que se encuentran en el grupo de 14 a 17 años. Este grupo reporta resultados también diversos. El motivo que muestra un aumento contundente es "el hogar necesita de su aportación económica" lo que podría estar vinculado con la mayor participación de estos niños y niñas en actividades fuera de las unidades económicas familiares respecto a los otros grupos de edad y la importancia de la aportación monetaria en función del aumento de la edad de los niños y las niñas.

No obstante, el motivo que mayor porcentaje presenta es "pagar sus propios gastos" con 29\%. Para muchas familias, que los hijos e hijas trabajen -sobre todo en etapas adolescentes- representa una forma de disminución en las erogaciones que se hacen por concepto de ropa, útiles escolares y artículos personales, con lo que en cierta forma los niños y las niñas complementan el ingreso familiar y disminuyen el costo de la manutención cotidiana (Alarcón, 2009).

Nuevamente el motivo "el hogar necesita de su trabajo" marca como importante con $26 \%$ de los encuestados. No es tan alto el porcentaje si se compara con el grupo de 10 a 13 años, lo cual resulta evidente ya que a mayor edad es más probable que se busque empleo fuera de las unidades económicas domésticas.

Tabla 4. Motivos por los que trabaja la población ocupada 14 a 17 años desplegada por sexo ${ }^{10}$

\begin{tabular}{lcccc}
\hline & Niños & Niñas & Total & $\%$ \\
\hline \hline $\begin{array}{l}\text { El bogar necesita de su } \\
\text { aportación económica }\end{array}$ & 232597 & 112870 & 345467 & $16 \%$ \\
$\begin{array}{l}\text { El bogar necesita de su } \\
\text { trabajo }\end{array}$ & 357577 & 197240 & 554817 & $26 \%$ \\
$\begin{array}{l}\text { Aprender un oficio } \\
\begin{array}{l}\text { Para pagar su escuela y } \\
\text { sus propiso gastos }\end{array}\end{array}$ & 228094 & 46906 & 275000 & $13 \%$ \\
No quiere ir a la escuela & 117220 & 213201 & 625400 & $29 \%$ \\
Otra razón & 127420 & 85415 & 212835 & $10 \%$ \\
Total & 1475107 & 677479 & 2152586 & $100 \%$ \\
\hline
\end{tabular}

Otra forma de analizar los motivos por los que trabajan los niños y niñas en México es abordarlo por sector de actividad. En el sector agropecuario por ejemplo, se tiene que $45 \%$ de los niños y niñas que trabajan lo hacen debido a que "el hogar

\footnotetext{
9 Elaboración propia con base en Encuesta Nacional de Ocupación y Empleo 2011, Módulo de Trabajo Infantil, INEGI (2012).

10 Elaboración propia con base en Encuesta Nacional de Ocupación y Empleo 2011, Módulo de Trabajo Infantil, INEGI (2012).
} 
necesita de su trabajo". Ello resulta lógico debido a que si bien hay una parte importante de este sector vinculado con la agricultura comercial y articulada al mercado internacional, en México la actividad agrícola aún se concentra en espacios de economías campesinas dirigidas hacia la auto subsistencia familiar, en las que el trabajo de todos los miembros de la familia es esencial (Tabla 5).

Tabla 5. Motivos por los que trabaja la población ocupada 5 a 17 años en el sector agropecuario desplegado por sexo ${ }^{11}$

\begin{tabular}{|c|c|c|c|c|}
\hline & Niños & Niñas & Total & $\%$ \\
\hline $\begin{array}{l}\text { El hogar necesita de su } \\
\text { aportación económica }\end{array}$ & 102195 & 12710 & 114905 & $13 \%$ \\
\hline $\begin{array}{l}\text { El bogar necesita de su } \\
\text { trabajo }\end{array}$ & 332437 & 69564 & 402001 & $45 \%$ \\
\hline Aprender un oficio & 158010 & 9516 & 167526 & $19 \%$ \\
\hline $\begin{array}{l}\text { Para pagar su escuela y } \\
\text { sus propios gastos }\end{array}$ & 87368 & 5617 & 92985 & $10 \%$ \\
\hline No quiere ir a la escuela & 44613 & 6336 & 50949 & $6 \%$ \\
\hline Otra razón & 55577 & 10706 & 66283 & $7 \%$ \\
\hline Total & 780200 & 114449 & 894649 & $100 \%$ \\
\hline
\end{tabular}

Para el caso de la industria y la construcción (Tabla 6), se muestra que 3 de cada 10 niños y niñas ocupados en este sector aducen que trabajan debido a que deben "pagar su escuela y sus gastos propios". Siguiéndole los motivos "el hogar necesita su trabajo" y "aprender un oficio" con 18\%. El sector industrial y la construcción son actividades que incluyen un sinnúmero de actividades, y lo que probablemente nos dicen los datos es que estos niños y niñas en su mayoría no pertenecen a hogares que a su vez funcionen como unidades económicas de producción ya que como se observa en el Tabla 6 , únicamente el 18\% de ellos contestó que el motivo por el que trabaja es que "el hogar necesita de su trabajo". Estos niños y niñas y podrían estar ocupados en pequeños talleres artesanales familiares.

Tabla 6. Motivos por los que trabaja la población ocupada de 5 a 17 años en el sector industria y construcción desplegada por sexo ${ }^{12}$

\begin{tabular}{|c|c|c|c|c|}
\hline & Niños & Niñas & Total & $\%$ \\
\hline $\begin{array}{l}\text { El bogar necesita de su } \\
\text { aportación económica }\end{array}$ & 63944 & 22647 & 86591 & $17 \%$ \\
\hline $\begin{array}{l}\text { El bogar necesita de } s u \\
\text { trabajo }\end{array}$ & 57605 & 35872 & 93477 & $18 \%$ \\
\hline Aprender un oficio & 75241 & 17176 & 92417 & $18 \%$ \\
\hline $\begin{array}{l}\text { Para pagar su escuela y } \\
\text { sus propios gastos }\end{array}$ & 120258 & 38749 & 159007 & $31 \%$ \\
\hline No quiere ir a la escuela & 27812 & 3069 & 30881 & $6 \%$ \\
\hline Otra razón & 28550 & 16618 & 45168 & $9 \%$ \\
\hline Total & 373410 & 134131 & 507541 & $100 \%$ \\
\hline
\end{tabular}

Los servicios y el comercio tienen una importante participación de niños y niñas. Los motivos que salen a la luz son "pagar la escuela y los propios gastos" con tres niños y niñas de cada diez que dijeron estar ocupados en este sector. Llama la atención que para este sector económico se esperaría que el motivo más importante fuera que el hogar necesita del trabajo de los niños y niñas, ya que por lo general las unidades domésticas funcionan como proveedoras de comercios y en algunos casos servicios. No obstante este motivo representa sólo el 22\%. Este porcentaje supera al resto de los niños y niñas que trabajan en otros sectores económicos, sin embargo no es el motivo que encabeza la lista. El sector servicios concentra sobre todo el trabajo de las niñas que están más vinculadas al trabajo doméstico en hogares ajenos. Ello podría estar explicando que el motivo "pagar la escuela y sus propios gastos" sea el motivo que encabeza la lista, pues como se vio líneas arriba, siete de cada diez niñas que trabajan lo hacen en el sector servicios y comercio.

11 Elaboración propia con base en Encuesta Nacional de Ocupación y Empleo 2011, Módulo de Trabajo Infantil, INEGI (2012).

12 Elaboración propia con base en Encuesta Nacional de Ocupación y Empleo 2011, Módulo de Trabajo Infantil, INEGI (2012). 
Tabla 7. Motivos por los que trabaja la población ocupada de 5 a 17 años en el sector comercio y servicios desplegados por sexo ${ }^{13}$

\begin{tabular}{|c|c|c|c|c|}
\hline & Niños & Niñas & Total & $\%$ \\
\hline $\begin{array}{l}\text { El bogar necesita de su } \\
\text { aportación económica }\end{array}$ & 102013 & 86923 & 188936 & $12 \%$ \\
\hline $\begin{array}{l}\text { El bogar necesita de su } \\
\text { trabajo }\end{array}$ & 153595 & 187721 & 341316 & $22 \%$ \\
\hline Aprender un oficio & 136012 & 68782 & 204794 & $13 \%$ \\
\hline $\begin{array}{l}\text { Para pagar su escuela y } \\
\text { sus propios gastos }\end{array}$ & 293195 & 223941 & 517136 & $33 \%$ \\
\hline No quiere ir a la escuela & 50795 & 15778 & 66573 & $4 \%$ \\
\hline Otra razón & 136456 & 125807 & 262263 & $17 \%$ \\
\hline Total & 872066 & 708952 & 1581018 & $100 \%$ \\
\hline
\end{tabular}

\section{Reflexiones finales}

La descripción que se ha realizado de los datos más recientes sobre el trabajo infantil en México deja varias reflexiones por delinear. En primer lugar, se presentan algunas líneas en cuanto al espacio familiar como la principal unidad en la que se organiza y materializa el trabajo o los ingresos de los miembros que la conforman, incluyendo a los niños y niñas. Este importante señalamiento teórico se comprueba en términos empíricos cuando en los datos aparecen como principales motivos que el hogar necesita ya sea del trabajo o de las aportaciones de los niños y las niñas ocupadas en México.

En este sentido, se observa al trabajo infantil como un fenómeno que se inserta en la lógica de las decisiones que se gestan al interior de los hogares, influenciados éstos por los condicionamientos de orden estructural. Como bien afirman los teóricos arriba mencionados, el trabajo infantil constituye una importante acción frente a la escases material y se comporta en esta misma dirección, es decir, cuando en los hogares no es suficiente el ingreso global para enfrentar los costos de manutención de los hijos e hijas, éstos tienden a salir al mercado de trabajo en busca de ingresos para sufragar sus gastos personales.

Igualmente se muestra en el análisis empírico que los hogares son al mismo tiempo espacios en donde se realizan actividades económicas, por lo que es en este espacio en que se hace uso de el recurso de individuos disponibles para la actividad económica. Tanto el número, como las características de quienes participan en el mercado de trabajo, depende en gran medida, de la composición de los grupos domésticos. Así sucede, cuando los niños y las niñas declaran que uno de los principales motivos por los que trabajan es porque el hogar necesita de su trabajo.

De ahí que sea perfectamente aplicable lo que los aportes teóricos establecen respecto a que las estrategias de sustento de los hogares se encuentran vinculadas con la movilización de la fuerza de trabajo. Éste es uno de los recursos fundamentales con que cuentan los hogares $y$, en este mismo sentido, se van configurando las formas de inserción laboral y social de los hijos e hijas.

Otra importante conclusión que surge de este ejercicio es que el motivo pagar los gastos propios tiene la mayor presencia en el sector comercio y servicios, donde el trabajo infantil es predominantemente femenino. Esto hace pensar varias cuestiones, en primer lugar, que la lógica de la unidades domésticas en el sector servicios es completamente distinta las que se vinculan con el agro en las que los gasto son socializados por todos los miembros de las unidades domésticas; por otra parte, que, por lo menos, en el sector comercio y servicios las niñas tienen la importante carga de género respecto a su auto manutención, viéndose en una desventaja respecto a los miembros varones del hogar. En este sentido se ratifica lo que presupone la teoría, acerca de que es en el ámbito doméstico donde también se gestan algunas contradicciones y se reproducen modelos sociales que puede perpetuar las desventajas.

Cabe señalar, también, que este tipo de acercamiento a la problemática del trabajo infantil desde el ámbito

13 Elaboración propia con base en Encuesta Nacional de Ocupación y Empleo 2011, Módulo de Trabajo Infantil, INEGI (2012). 
de los hogares tiene algunas debilidades ya que difícilmente puede dar cuenta de los contextos estructurales a los que se enfrentan las familias $y$ dice muy poco respecto a la forma en que se toman las decisiones. Pero sobre todo no da luces sobre el papel o la agencia de los propios niños y niñas en este fenómeno social. Sin embargo se constituye como un ejercicio que puede guiar los primeros acercamientos al conocimiento sobre la práctica del trabajo infantil en México.

No cabe duda que un porcentaje significativo de niños y niñas que trabajan en México están fungiendo el papel de proveedores en sus hogares a temprana edad. Otro importante porcentaje se hace cargo de sus propios gastos, y en general los niños y niñas que dicen estar ocupados asumen roles familiares y sociales que no deberían asumir a edades tempranas, pues en muchos de los casos eso significa sacrificar un crecimiento saludable y apropiado para hacerle frente al futuro.

Finalmente, es de suma importancia reconocer que las problemáticas que atañen a la infancia, entre ellas el trabajo y las condiciones en que se realiza, constituyen una extensión de los problemas de la sociedad en general. En la medida que la infancia aprenda y reproduzca roles económicos y de género influenciados por procesos macrosociales se les estará empujando a acostumbrarse y a naturalizar relaciones laborales de desventaja y subordinación absoluta. En esa misma medida se irá moldeando en la sociedad a los individuos desde las primeras etapas de la vida.

\section{Referencias}

Alarcón, W. (2009) De la explotación a la esperanza. Ensayos sobre trabajo infantil en América Latina, Proyecto SolidarioOIT, España.

Barsotti, C. A. (1981) "La organización social de la reproducción de los agentes sociales, las unidades familiares y sus estrategias". En: Demografía y Economía, XV (2). El Colegio de México, México.

Bertaux, D. (1996) "Historias de casos de familias como método para la investigación de la pobreza". En: Revista de Sociedad, Cultura y Política, Vol. 1 (1). Buenos Aires.

García, B. \& De Oliveira O. (2006) Las familias en el México metropolitano: visiones femeninas y masculinas, El Colegio de México, México.

González de la Rocha, M. (coord.) (2009) Procesos domésticos y vulnerabilidad. Perspectivas antropológicas de los hogares con Oportunidades. Publicaciones de la casa Chata, CIESAS, México

INEGI (2012) Encuesta Nacional de Ocupación y Empleo 2011, Módulo de Trabajo Infantil. México.

Intervida (2008) Vidas explotadas. La explotación laboral infantil. Fundación Intervida. Barcelona, España.

Macri, M. (2011) "Trabajo infantil y familia: los estudios sociológicos sobre la familia como marco interpretativo para el trabajo infantil intrafamiliar". En: Fodor, S. (Coord.) Problemáticas Infanto juveniles. ERREPAR S.A. B.A. Argentina.

Sáenz, A. \& Di Paula, J. (1981) "Precisiones teórico-metodológicas sobre la noción de estrategias de existencia". En: Demografía y Economía, XV (2). El Colegio de México, México.

Torrado, S. (1981) "Sobre el concepto de estrategias familiares de vida y proceso de reproducción de la fuerza de trabajo. Notas teórico-metodológicas". En: Demografía y Economía, XV (2). El Colegio de México, México.

Valdés, X. \& Acuña, M. (1981) "Precisiones metodológicas sobre las estrategias de supervivencia”. En: Demografía y Economía, XV (2). El Colegio de México, México.

Villasmil, M. C. (1998) "Apuntes teóricos para la discusión sobre el concepto de estrategias en el marco de los estudios de población". En: Estudios Sociológicos. México, El Colegio de México, Centro de Estudios Sociológicos, Vol. 16 (46) (ene.-abr. 1998). México. 